\title{
Cytoprotective effect exerted by geraniin in HepG2 cells is through microRNA mediated regulation of $\mathrm{BACH}-1$ and $\mathrm{HO}-1$
}

\author{
Hoda Aayadi ${ }^{1}$, Smriti P.K. Mittal ${ }^{1, \dagger}$, Anjali Deshpande ${ }^{2}$, Makarand Gore ${ }^{2}$ E Saroj S. Ghaskadbi ${ }^{1, *}$ \\ ${ }^{1}$ Department of Zoology, Savitribai Phule Pune University, ${ }^{2}$ AJ Organica Pvt. Ltd., Pune - 411007, India
}

\begin{abstract}
Geraniin, a hydrolysable tannin, used in traditional medicine in Southeast Asia, is known to exhibit various biological activities. As an antioxidant it is known to up-regulate phase II enzyme Heme oxygenase-1 (HO-1). However its mechanism is not clearly understood. Nuclear factor erythroid-derived 2 related factor 2 (Nrf-2) is transcriptionally up-regulated by Extracellular signal-regulated kinase (ERK) $1 / 2$ and retained in nucleus due to inactivated Glycogen synthase kinase 3 beta (GSK-3ß). Geraniin additionally down-regulates expression of microRNA 217 and 377 (miR-217 and miR-377) which target HO-1 mRNA. Expression of BTB and CNC homolog 1 (BACH-1), another regulator of HO-1, is also down-regulated by up-regulating microRNA 98 (miR-98), a negative regulator of BACH-1. Thus, geraniin up-regulates HO-1 expression both through activating its positive regulator Nrf-2 and by down-regulating its negative regulator $\mathrm{BACH}-1$. Up-regulation of HO-1 also confers protection to HepG2 cells from tertiary butyl hydroperoxide (TBH) induced cytotoxicity. [BMB Reports 2017; 50(11): 560-565]
\end{abstract}

\section{INTRODUCTION}

Reactive oxygen species (ROS) are generated as byproducts of metabolism or due to extracellular stress. Though, low levels of ROS are necessary for cells as activators of signal transduction pathways, excessive generation of ROS leads to oxidative stress (OS) where biological molecules such as proteins, lipids, and DNA are damaged ultimately resulting in cell death via apoptosis or necrosis (1). OS also plays an important role in the pathogenesis and progression of different diseases like cancer, diabetes, liver cirrhosis, etc. $(2,3)$. To overcome damaging effects of ROS, under normal conditions,

*Corresponding author. Tel: +91-9850993508; Fax: +91-2025690617; E-mail: ssg@unipune.ac.in

${ }^{\dagger}$ Present address: Department of Biotechnology, Savitribai Phule Pune University, Pune - 411007, India

https://doi.org/10.5483/BMBRep.2017.50.11.060

Received 10 April 2017, Revised 10 May 2017, Accepted 7 June 2017

Keywords: Antioxidants, Geraniin, HO-1, miRNAs, Nrf-2 cells activate their defense mechanisms through endogenous antioxidant enzymes and molecules. However, to ensure sufficient levels of antioxidants, very often natural antioxidant molecules are used as dietary supplements $(4,5)$.

Geraniin is a hydrolyzable tannin which has a unique dehydrohexahydroxydiphenoyl moiety and is widely distributed amongst dicotyledonous plants. There have been several studies on geraniin, either as a plant extract or pure compound, where it has been shown to exhibit strong antioxidative potential $(6,7)$ as well as anticancer, antiviral, antihypertensive and antihyperglycaemic (8-12) activities.

Cellular antioxidant defense system consists of first line enzymes such as superoxide dismutase (SOD), catalase (CAT) and phase II detoxification enzymes such as glutathione S-transferases, glutamate-cysteine ligase, $\mathrm{NAD}(\mathrm{P}) \mathrm{H}$ : quinine oxidoreductase, glutathione peroxidase (GPx) and HO-1. Most of the supplementary antioxidants used are known to activate HO-1. Induction of HO-1 requires Nrf-2 activation, its translocation to nucleus and binding to cis-acting enhancer element in promoter of HO-1, known as ARE (13). Among other regulatory factors binding to ARE motif is $\mathrm{BACH}-1$ which is a transcriptional repressor competing with $\mathrm{Nrf}-2$ for binding to $\operatorname{ARE}(14,15)$. $\mathrm{HO}-1$ and its negative regulator $\mathrm{BACH}-1$ are also regulated by specific microRNAs (miRNAs).

Geraniin is known to up-regulate the expression of HO-1 by activating PI3K/AKT and MAPK signaling pathways (16). In the present study, we demonstrate that up-regulation of $\mathrm{HO}-1$ is by activation of upstream regulators of Nrf-2, namely, GSK-3 $\beta$ and ERK, and on levels of miRNAs involved in regulation of both $\mathrm{BACH}-1$ and $\mathrm{HO}-1$. We have also shown that this up-regulation of HO-1 by geraniin confers cytoprotection to HepG2 cells against TBH induced toxicity by using tin protoporphyrin (SnPP), specific inhibitor of HO-1.

\section{RESULTS}

Geraniin exhibits protective effect against TBH-induced cytotoxicity in HepG2 cells

Viability of HepG2 cells exposed to different concentrations of geraniin for $24 \mathrm{hrs}$ was checked. It did not change significantly between $1-25 \mu \mathrm{M}$ whereas at 50 and $100 \mu \mathrm{M}$ concentration it was significantly reduced (Fig. 1A). Therefore, the concentration range of 1-25 $\mu \mathrm{M}$ was used for further experiments. To check

ISSN: 1976-670X (electronic edition)

Copyright (c) 2017 by the The Korean Society for Biochemistry and Molecular Biology

(c) This is an open-access article distributed under the terms of the Creative Commons Attribution Non-Commercial License (http://creativecommons.org/licenses/by-nc/4.0) which permits unrestricted non-commercial use, distribution, and reproduction in any medium, provided the original work is properly cited. 


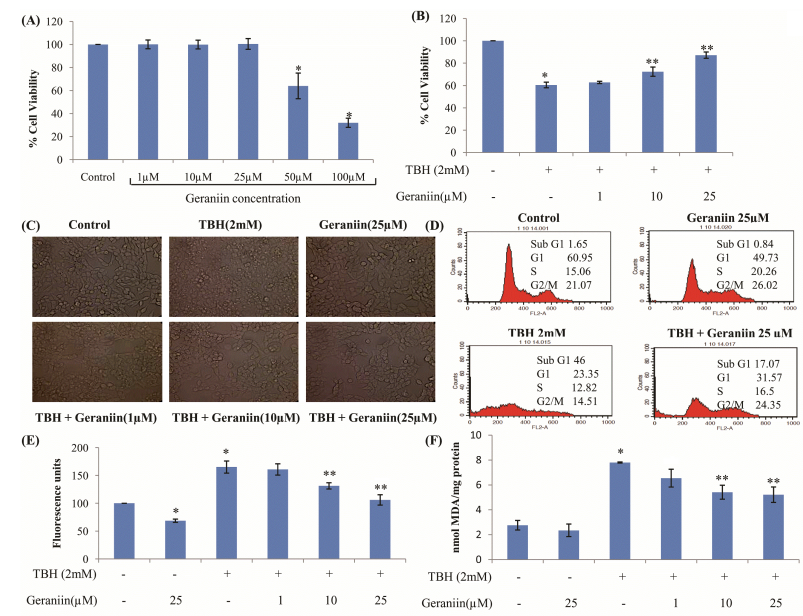

Fig. 1. Geraniin exhibits protective effect against $\mathrm{TBH}$-induced cytotoxicity in HepG2 cells. (A) HepG2 cells were treated with various concentrations of geraniin for $24 \mathrm{~h}$, and the viability was measured by MTT assay. Data shown represent the mean values of three experiments \pm SE. $* P<0.05$ vs, control. (B) Cells were pretreated with different concentrations of geraniin $(0,1,10$, and $25 \mu \mathrm{M})$ for $2 \mathrm{hrs}$, followed by exposure to TBH (2 mM). Cell viability was assessed by MTT assay. Data shown represent the mean values of three experiments \pm SE. ${ }^{* P}<0.05$ vs. control. $* * \mathrm{P}<0.05$ vs. TBH $(2 \mathrm{mM})$. (C) Cell morphology was observed under inverted microscope. (D) Cell cycle analysis was done in presence and absence of geraniin $(25 \mu \mathrm{M})$. (E) Inhibition of intracellular ROS generated and (F) lipid peroxidation on TBH treatment in presence of geraniin. Data shown represent the mean values of three experiments $\pm \mathrm{SE}$. ${ }^{* P}<0.05$ vs. control. $* * \mathrm{P}<$ 0.05 vs. TBH $(2 \mathrm{mM})$.

protective effect of geraniin against TBH-induced OS, cells were pre-treated with geraniin for $2 \mathrm{hrs}$ and then exposed to TBH for 3 hrs. Pretreatment with 10 and $25 \mu \mathrm{M}$ geraniin significantly protected cells against TBH-induced cytotoxicity (Fig. 1B). Morphological studies also confirmed the same. TBH treated cells were shrunk, rounded and stressed whereas incubation with 10 and $25 \mu \mathrm{M}$, but not $1 \mu \mathrm{M}$ geraniin for $2 \mathrm{hrs}$ restored cell morphology effectively (Fig. 1C). To further confirm these results, cell cycle analysis was performed. As seen in Fig. 1D, in cells treated with geraniin alone, the cell cycle profile remained unaltered, whereas in cells treated with $2 \mathrm{mM}$ TBH for $3 \mathrm{hrs}, 46 \%$ of cell population was apoptotic. In cells treated with geraniin followed by exposure to $\mathrm{TBH}$, apoptosis was reduced significantly $(\mathrm{P}<0.05)$ to $17 \%$.

TBH is known to produce ROS and induce lipid peroxidation in cells. Therefore, amount of ROS generated in cells was evaluated by using non-fluorescent DCFH-DA dye. Significant increase in ROS in TBH treated cells was observed which in presence of geraniin was significantly reduced (Fig. 1E). Treatment of cells with TBH increased lipid peroxidation to $7.79 \pm 0.04$ nmols MDA/mg protein (Fig. 1F) and in cells treated with geraniin this was significantly reduced.

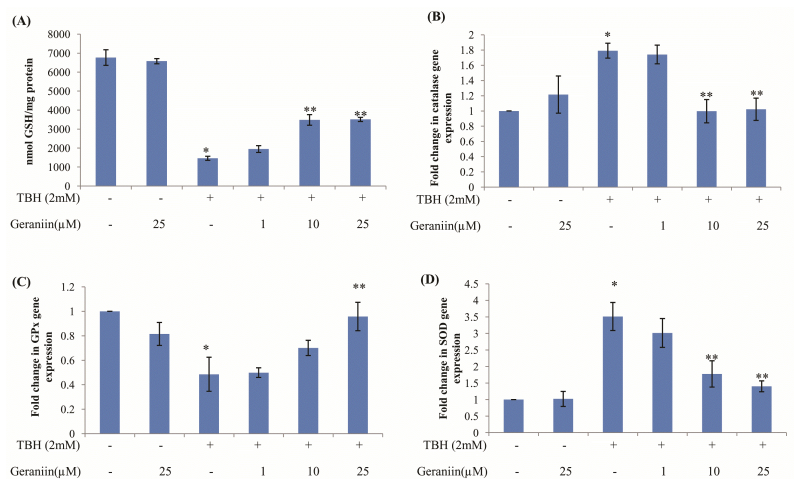

Fig. 2. Altered antioxidant enzyme gene expression and GSH level in HepG2 cells treated with $\mathrm{TBH}$ is restored in presence of Geraniin. (A) Intracellular GSH level was determined in terms of nmol GSH/mg protein. (B) CAT (C) GPx and (D) SOD gene expression was measured in HepG2 cells pretreated with geraniin $(0,1,10$, and $25 \mu \mathrm{M})$ for 2 hrs and exposed to TBH $(2 \mathrm{mM})$ for 3 hrs. Data shown represent the mean values of three experiments \pm SE. ${ }^{* P}<0.05$ vs. control. ${ }^{* * P}<0.05$ vs. TBH (2 $\mathrm{mM})$.

\section{Altered antioxidant enzyme gene expression and GSH level in HepG2 cells treated with TBH is restored in presence of geraniin}

Glutathione, major antioxidant of the cell was found to be reduced significantly in cells exposed to $\mathrm{TBH}$, which was effectively restored by up to $40 \%$ in presence of higher concentration of geraniin (Fig. 2A).

Expression of antioxidant enzymes such as CAT, GPx and SOD was measured using quantitative real-time PCR (qRT-PCR) and by biochemical assays. Exposure to $\mathrm{TBH}$, significantly increased CAT and SOD expression to $1.79 \pm 0.09$ and 3.32 \pm 0.58 fold respectively and decreased GPx expression to $0.48 \pm 0.13$ fold. Treatment with $25 \mu \mathrm{M}$ geraniin significantly restored the level of all antioxidant enzymes. Geraniin by itself did not alter level of antioxidant enzymes (Fig. 2B-D).

\section{Geraniin increases HO-1 expression through Nrf-2 nuclear translocation}

Activation of HO-1, by geraniin, was checked both by western blot and qRT-PCR. Cells were treated with $25 \mu \mathrm{M}$ geraniin for different time intervals and expression of HO- 1 was evaluated. $\mathrm{HO}-1$ increased in a time-dependent manner with maximum expression observed at 90 min both by western blot analysis and qRT-PCR (Fig. 3A-C). To confirm that cytoprotective effect of geraniin against TBH is indeed due to $\mathrm{HO}-1$ expression, cells were treated with SnPP which is a potent competitive inhibitor of HO-1. SnPP significantly suppressed cell protection exerted by geraniin indicating involvement of up-regulated $\mathrm{HO}-1$ in conferring cytoprotection (Fig. 3D). Since Nrf-2 is a known potent regulator of $\mathrm{HO}-1$, its localization was determined by immunostaining. We observed that treatment 


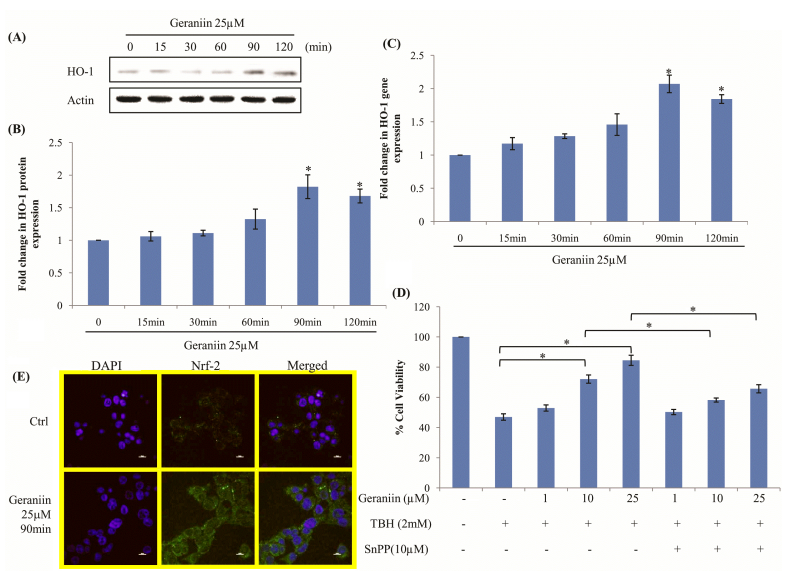

Fig. 3. Geraniin increases HO-1 expression by Nrf-2 nuclear translocation in cells. Cells were treated with $25 \mu \mathrm{M}$ geraniin at different time points and HO-1 expression was determined by (A) Western blot analysis, (B) its densitogram analysis and (C) qRT-PCR. Data shown represent the mean values of three experiments \pm SE. $* \mathrm{P}<0.05$ vs. Control. Cells treated with Geraniin (D) To confirm involvement of $\mathrm{HO}-1$ in protection caused by Geraniin, cells were treated with 1, 10 and $25 \mu \mathrm{M}$ of geraniin and $10 \mu \mathrm{M}$ SnPP, and were subsequently exposed to TBH $(2 \mathrm{mM})$ for $3 \mathrm{hrs}$. Cell viability was determined by MTT. Data shown represent the mean values of three experiments \pm $\mathrm{SE}$. $* \mathrm{P}<0.05$. (E) $\mathrm{Nrf}-2$ a positive regulator of $\mathrm{HO}-1$ was detected by immunolocalisation in control and $25 \mu \mathrm{M}$ geraniin treated cells for 90 mins.

with geraniin significantly up-regulates Nrf-2 which translocates to nucleus (Fig. 3E).

\section{Geraniin phosphorylates upstream regulators of $\mathrm{Nrf}-2$ namely ERK1/2 and GSK-3 $\beta$}

MAPKs such as ERK1/2, JNK and P38 are known to be involved in activation of Nrf-2 pathway (17). Under OS, these get phosphorylated and activate Nrf-2 cascade. Treatment with geraniin led to time-dependent induction of only ERK phosphorylation (Fig. 4A). To confirm the involvement of activated ERK in protection exhibited by geraniin, an inhibitor against ERK (PD98059, 2'-amino-3'-methoxyflavone) was used and cell protection was checked. Inhibition of ERK effectively repressed cytoprotection caused by geraniin confirming its involvement in induction of HO-1 through Nrf-2 (Fig. 4B).

GSK-3 $\beta$ is known negative regulator of Nrf-2. In presence of geraniin, increase in pGSK-3 $\beta$ (ser9) was observed leading to its inactivation (Fig. 4C and D). Inactivation of GSK-3 $\beta$ leads to retention of Nrf-2 in the nucleus favouring induction of HO-1.

\section{Geraniin regulates miRNA targets of $\mathrm{HO}-1$ and $\mathrm{BACH}-1$}

Another important negative regulator of $\mathrm{HO}-1$ is $\mathrm{BACH}-1$ which competes with $\mathrm{Nrf}-2$ in binding to ARE motif. The expression of $\mathrm{BACH}-1$ was significantly reduced at $90 \mathrm{~min}$. Expression of miR-98 a known negative regulator of $\mathrm{BACH}-1$

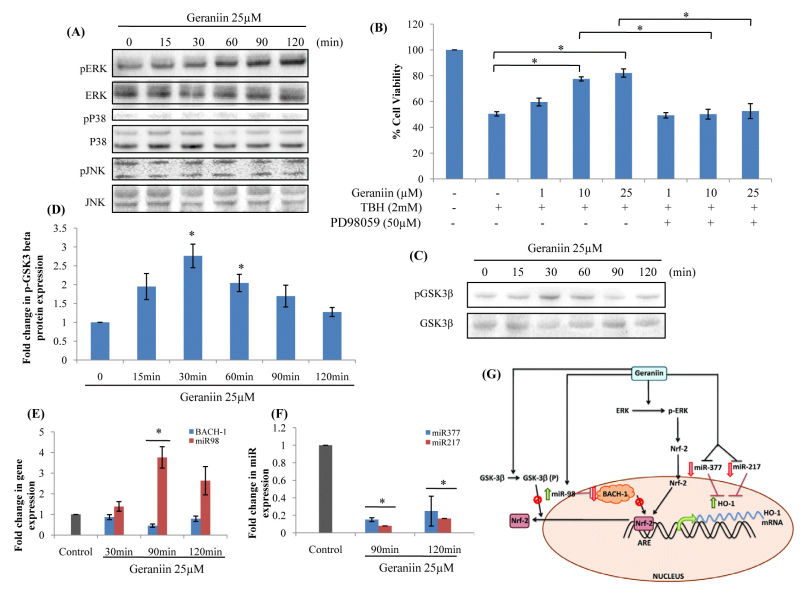

Fig. 4. Geraniin activates $\mathrm{HO}-1$ by regulating upstream targets. (A) Western blot analysis of pERK, pJNK and pP38 MAPKs in HepG2 cells subjected to $25 \mu \mathrm{M}$ geraniin at various time points. (B) To confirm the role of pERK MAPK in protection offered by geraniin, cells were treated with $0,1,10,25 \mu \mathrm{M}$ geraniin and $50 \mu \mathrm{M}$ PD98059, an inhibitor of ERK1/2, and were subsequently exposed to TBH $(2 \mathrm{mM})$ for $3 \mathrm{hrs}$. Cell viability was determined by MTT. Data shown represent the mean values of three experiments \pm SE. (C) Western blot analysis of pGSK-3 $\beta$ and (D) its densitogram analysis, in HepG2 cells subjected to $25 \mu \mathrm{M}$ geraniin at indicated time periods. (E) qRT-PCR analysis of $\mathrm{BACH}-1$ and its negative regulator, miR-98 expression and (F) negative regulators of $\mathrm{HO}-1$, miR-377 and miR-217 expression in HepG2 cells treated with 25 $\mu \mathrm{M}$ geraniin at indicated time periods. Data shown represent the mean values of three experiments \pm SE. ${ }^{*} \mathrm{P}<0.05$ vs. Control. (G) Proposed molecular mechanism of cytoprotective role of geraniin in HepG2 cells.

was checked. We indeed observed, increase in miR-98 expression at 90 mins, which led to decrease in $\mathrm{BACH}-1$ expression (Fig. 4E).

miR-377 and miR-217 are negative regulators of $\mathrm{HO}-1$ which affect gene expression post-transcriptionally by interacting with HO-1 mRNA and inhibiting translation. Significant decrease in expression of both miR-377 and miR-217 was observed in presence of geraniin (Fig. 4F).

\section{DISCUSSION}

Geraniin is a dehydroellagitannin, present in many medicinal herbs known to exhibit different biological activities among which antioxidant activity has been widely studied (6). Cytoprotective effects of geraniin against OS has been shown previously in different cell types $(16,18,19)$. In a recent study geraniin has shown to exhibit cytoprotective effect by reducing $\mathrm{H}_{2} \mathrm{O}_{2}$-induced cell death in vitro by activating PI3K/AKT and ERK $1 / 2$ pathways and Nrf-2 signaling (16). We initially confirmed radical scavenging property of geraniin using standard radical scavenging assays (Supplementary Fig. 1) and observed that it reduced formation of lipid peroxides, restored 
protein sulphydryl groups and also inhibited formation of single/double strand breaks in plasmid DNA against OS in a concentration-dependent manner (Supplementary Fig. 2).

In cells treated with geraniin, TBH induced cytotoxicity, ROS production and lipid peroxidation was significantly reduced. TBH treatment led to increase in antioxidant defense enzymes namely SOD and CAT, while GPx was decreased. Transcripts of all these enzymes were restored on geraniin treatment. Geraniin by itself did not activate any one of these enzymes. GSH, a major redox buffer of cell was observed to be decreased on TBH treatment and this was prevented significantly in presence of Geraniin. All these results, demonstrate a strong antioxidant potential of geraniin.

HO-1, an important phase II antioxidant enzyme was strongly up-regulated in presence of geraniin and its involvement in cytoprotective effect against TBH was confirmed by using SnPP, competitive inhibitor of HO-1 activity. Induction of $\mathrm{HO}-1$ was due to up-regulation of Nrf-2, a known positive regulator of HO-1. Since, MAP kinases are known to be involved in activation of Nrf-2, the expression of p38, JNK and ERK was checked and only ERK was found to be phosphorylated in presence of geraniin similar to a previous report (16). This was further reconfirmed by using ERK inhibitor (PD98059), in whose presence cytoprotection offered by geraniin was significantly reduced.

GSK-3 $\beta$ normally promotes degradation of Nrf-2. If GSK-3 $\beta$ is inactivated, Nrf-2 is not degraded and accumulates in the nucleus. We indeed observed increase in pGSK-3 $\beta$ (Ser 9) on geraniin treatment which inactivates the enzyme leading to retention of $\mathrm{Nrf}-2$ in the nucleus which was confirmed by immunostaining. Thus, we have shown that both activation of ERK and inactivation of GSK-3 $\beta$ are important for up-regulation of $\mathrm{Nrf}-2$ on geraniin treatment, which then leads to HO-1 activation.

miR-217 and miR-377 directly regulate HO-1 expression (20). Both these miRNAs were significantly reduced in presence of geraniin which helped in enhanced translation of HO-1 mRNA.

Another regulator of $\mathrm{HO}-1$ is $\mathrm{BACH}-1$, which competes with $\mathrm{Nrf}-2$ in binding to ARE motif and represses downstream gene expression. On its release form ARE motif, it allows Nrf-2 to bind to its enhancer sequence and activate gene expression (15). We observed that in the cells treated with geraniin, $\mathrm{BACH}-1$ expression was significantly reduced. $\mathrm{BACH}-1$, itself is also known to be regulated negatively by a number of miRNAs namely, let-7b, Let-7c, miR-196, miR-98. Out of these we found only miR-98 to be significantly increased when $\mathrm{BACH}-1$ expression was significantly reduced.

Present study thus confirms cytoprotective effect of geraniin against TBH induced cytotoxicity in HepG2 cells via HO-1 induction. HO-1 activation by geraniin is brought about both by up-regulating its positive regulator $\mathrm{Nrf}-2$ and down-regulating its negative regulator $\mathrm{BACH}-1$ and $\mathrm{HO}-1$ specific microRNAs (miR-217 and miR-377) (Fig. 4G).

\section{MATERIALS AND METHODS}

\section{Chemicals}

Dulbecco's modified essential media (DMEM), Foetal Bovine Serum (FBS), trypsin and Penicillin-streptomycin were purchased from Gibco. TBH, 2-thiobarbituric acid (TBA), 2', $7^{\prime}$ dichlorofluorescein diacetate (DCFH-DA) and 3-(4,5-dimethylthiazol-2-yl)-2,5-diphenyltetrazolium bromide (MTT) were purchased from Sigma Aldrich, USA. Reduced glutathione, potassium phosphate, sodium carbonate, trichloroacetic Acid (TCA) and 5,5'-dithio-bis (2 nitrobenzoic acid) (DTNB) were obtained from Sisco Research Laboratory, India. Other common chemicals used in our studies were of the highest quality, commercially available from local suppliers. Geraniin (purity of $>90 \%$ ) was procured from AJ organic Pvt. Ltd., Pune, India.

\section{Cell culture}

HepG2 cell line was procured from National Centre for Cell Science, Pune, India. These cells were grown in DMEM supplemented with $10 \%(\mathrm{v} / \mathrm{v})$ FBS, $100 \mathrm{U} / \mathrm{ml}$ penicillin and $100 \mu \mathrm{g} / \mathrm{ml}$ streptomycin, in humidified atmosphere with $5 \%$ $\mathrm{CO}_{2}$ at $37^{\circ} \mathrm{C}$.

\section{Cell viability assay and morphology examination}

$2 \times 10^{4}$ cells were seeded per well in 96 well plate and incubated at $37^{\circ} \mathrm{C}$ in $\mathrm{CO}_{2}$ incubator for $48 \mathrm{hrs}$. Cells were then exposed to different concentrations of geraniin $(1,10,25,50$ and $100 \mu \mathrm{M})$ and $\mathrm{TBH}$. Cell viability was determined using MTT assay (21). Morphological changes were observed under a microscope, and images were taken with an Olympus digital camera (Olympus, Japan).

\section{Cell cycle analysis}

Control and treated cells were washed with $1 \mathrm{X}$ phosphate buffered saline (1X PBS) $(137 \mathrm{mM} \mathrm{NaCl}, 2.7 \mathrm{mM} \mathrm{KCl}, 10 \mathrm{mM}$ $\mathrm{Na}_{2} \mathrm{HPO}_{4}, 2 \mathrm{mM} \mathrm{KH_{2 }} \mathrm{PO}_{4}$ ) and fixed at $4^{\circ} \mathrm{C}$ for 2 hrs using $70 \%$ ethanol. The single cell suspension was then treated with RNase A $(100 \mathrm{mg} / \mathrm{ml})$ at $37^{\circ} \mathrm{C}$ for 30 mins followed by staining with $50 \mathrm{mg} / \mathrm{ml}$ propidium iodide (22). Stained cells were analyzed with FACSCanto, using cell quest software (BD, USA). Cell cycle profile was obtained by analyzing 10,000 cells per sample.

\section{Measurement of TBARS}

Lipid peroxidation was performed following the protocol described by Ohkawa et al., (23) with slight modification. In brief, $1.5 \times 10^{6}$ cells were seeded in $60 \mathrm{~mm}$ plates and incubated at $37^{\circ} \mathrm{C}$ for $48 \mathrm{hrs}$. Cells were then treated with geraniin $(1,10$ and $25 \mu \mathrm{M})$ for 2 hrs, followed by TBH exposure for $3 \mathrm{hrs}$. These were washed with 1X PBS, collected and homogenized in $100 \mu \mathrm{l}$ protein extraction buffer. $600 \mu \mathrm{l}$ TBA reagent $(0.8 \%(\mathrm{w} / \mathrm{v})$ TBA in $20 \%$ TCA) was added to each tube and vortexed. The reaction mixture was incubated at 
$95^{\circ} \mathrm{C}$ for 20 mins and reaction was terminated by keeping tubes on ice. Reaction mixture was centrifuged at 10,000 rpm for 10 mins and the absorbance of the total thiobarbituric reactive substances (TBARS) in supernatant was measured fluorometerically at Ex/Em wave length of 535/585 nm and expressed as nmoles of malondialdehyde (MDA) equivalents formed/mg protein.

\section{ROS measurement}

$1 \times 10^{4}$ cells were seeded per well in 96 well plate and after $48 \mathrm{hrs}$ incubation, they were treated with geraniin (1, 10 and $25 \mu \mathrm{M})$ for 2 hrs. Cells were then stained with $10 \mathrm{mM}$ DCFH-DA in serum free DMEM for 30 mins in the dark. Cells were washed twice with serum free DMEM and treated with 2 $\mathrm{mM}$ TBH for 3hrs. Fluorescence was recorded at an Ex/Em wavelength of $485 / 530 \mathrm{~nm}$ (24).

\section{Glutathione assay}

Glutathione assay was carried out using DTNB (25). $1.5 \times 10^{6}$ cells were seeded in $60 \mathrm{~mm}$ plates, incubated at $37^{\circ} \mathrm{C}$ for 48 hrs and treated with geraniin $(1,10$ and $25 \mu \mathrm{M})$ for 2 hrs followed by exposure to TBH for 3 hrs. Cells were then harvested and lysed. Proteins were transferred to fresh tubes followed by sulfosalicycilc acid addition. To each sample DTNB was added and the rate of its conversion to colored product was measured spectrophotometrically at $412 \mathrm{~nm}$ using ELISA reader. A standard curve was plotted using different concentrations of GSH.

\section{RNA Isolation and qRT-PCR amplification}

$1 \times 10^{6}$ cells were seeded in 6 well plate and incubated at $37^{\circ} \mathrm{C}$ for $48 \mathrm{hrs}$. They were then treated with geraniin for $2 \mathrm{hrs}$ followed by TBH exposure for $3 \mathrm{hrs}$. Cells were then washed with ice-cold PBS and RNA was isolated using TRIzol reagent. It was precipitated using isopropanol and dissolved in Diethyl pyrocarbonate (DEPC) treated water. After quantification, $2 \mu \mathrm{g}$ RNA was used for reverse transcription using cDNA synthesis kit (Thermo scientific, USA) according to manufacturers instruction. Resulting cDNA was used for qRT-PCR amplification (Step One Plus, Applied Biosystems, USA) using gene specific primers and actin was used as an internal control. From the same RNA using stem-loop primers, specific for microRNA, CDNA was synthesized and qRT-PCR was performed using miRNA-specific primers (26) (Supplementary Fig. 3).

\section{Western blot analysis}

HepG2 cells were harvested and washed with 1X PBS and lysed in $20 \mathrm{mM}$ Tris-Cl buffer $(\mathrm{pH}$ 7.4) containing protease inhibitor cocktail and $1 \mathrm{mM}$ phenylmethylsulfonyl fluoride (PMSF). Protein concentration was estimated using Lowry method and $40 \mu \mathrm{g}$ protein from each sample was resolved on $12 \%$ SDS-PAGE, followed by transfer onto a polyvinylidene difluoride (PVDF) membrane. Membrane was blocked with $5 \%(\mathrm{w} / \mathrm{v})$ bovine serum albumin or non-fat dry milk for 90 mins, incubated with the primary antibodies against p-JNK, p-p38, p-ERK and total ERK (Cell Signaling Technology, USA), total JNK, total p38 (Santa Cruz, USA), followed by incubation with peroxidase-conjugated secondary antibody for $1 \mathrm{hr}$ and finally detection by Optiblot ECL detect kit (Abcam, UK) using ChemiDoc (BioRad, US).

\section{Immunofluorescence assay}

$5 \times 10^{5}$ cells were cultured on cover slips in 6 well plate for $24 \mathrm{hrs}$ and incubated in presence or absence of geraniin for 90 mins. Cells were then washed with 1X PBS, fixed with Methanol:Acetone (3:1) and blocked with blocking buffer (3\% BSA, $0.3 \%$ Triton X-100 in 1X PBS) over night. Cells were incubated with rabbit anti-Nrf-2 antibody for $1 \mathrm{hr}$ followed by incubation with Alexa Fluor 488-labeled goat anti-rabbit IgG secondary antibody. Finally they were washed thoroughly, stained with 4',6-diamidino-2-phenylindole (DAPI) and cover slips were mounted with Vectashield (Vector Laboratories, USA).

\section{Statistical analysis}

Throughout the text, data are expressed as the mean \pm SE of at least three independent experiments. To determine statistical significance among different groups, one-way analysis of variance (ANOVA), followed by post-hoc test was performed using SPSS 19.0 software. $\mathrm{P}<0.05$ was considered as statistically significant.

\section{ACKNOWLEDGEMENTS}

This work was supported by University Grant CommissionCentre for Advance Studies (UGC-CAS) [F-5-2/2005(SAP-II)], Government of India, Department of Science and TechnologyPromotion of University Research and Scientific Excellence (DST-PURSE) [GOI-A-670], Government of India, India. HA was recipient of fellowship from Indian Council for Cultural Relations (ICCR).

\section{CONFLICTS OF INTEREST}

The authors have no conflicting interests.

\section{REFERENCES}

1. Martindale JL and Holbrook NJ (2002) Cellular response to oxidative stress: signaling for suicide and survival. J Cell Physiol 192, 1-15

2. Dalle-Donne I, Rossi R, Colombo R, Giustarini D and Milzani A (2006) Biomarkers of oxidative damage in human disease. Clin Chem 52, 601-623

3. Valko M, Leibfritz D, Moncol J, Cronin MT, Mazur M and Telser J (2007) Free radicals and antioxidants in normal physiological functions and human disease. Int J Biochem Cell Biol 39, 44-84

4. Droge W (2002) Free radicals in the physiological control 
of cell function. Physiol Rev 82, 47-95

5. Cadenas E (1997) Basic mechanisms of antioxidant activity. Biofactors 6, 391-397

6. Thitilertdecha N, Teerawutgulrag A, Kilburn JD and Rakariyatham N (2010) Identification of major phenolic compounds from Nephelium lappaceum L. and their antioxidant activities. Molecules 15, 1453-1465

7. Londhe JS, Devasagayam TP, Foo LY and Ghaskadbi SS (2008) Antioxidant activity of some polyphenol constituents of the medicinal plant Phyllanthus amarus Linn. Redox Rep 13, 199-207

8. Wongnoppavich A, Kanjana J and Seewaboon S (2009) Triphala: The Thai traditional herbal formulation for cancer treatment. Songklanakarin J Sci Technol 31, 139-149

9. Notka F, Meier GR and Wagner R (2003) Inhibition of wild-type human immunodeficiency virus and reverse transcriptase inhibitor-resistant variants by Phyllanthus amarus. Antiviral Res 58, 175-186

10. Yang CM, Cheng HY, Lin TC, Chiang LC and Lin CC (2007) The in vitro activity of geraniin and 1,3,4,6-tetra-Ogalloyl-beta-D-glucose isolated from Phyllanthus urinaria against herpes simplex virus type 1 and type 2 infection. J Ethnopharmacol 110, 555-558

11. Lin SY, Wang CC, Lu YL, Wu WC and Hou WC (2008) Antioxidant, anti-semicarbazide-sensitive amine oxidase, and anti-hypertensive activities of geraniin isolated from Phyllanthus urinaria. Food Chem Toxicol 46, 2485-2492

12. Palanisamy UD, Ling LT, Thamilvaani $M$ and David A (2011) Rapid isolation of geraniin from Nephelium lappaceum rind waste and its anti-hyperglycemic activity. Food Chem 127, 21-27

13. Wasserman WW and Fahl WE (1997) Functional antioxidant responsive elements. Proc Natl Acad Sci U S A 94, 5361-5366

14. Dhakshinamoorthy S, Jain AK, Bloom DA and Jaiswal AK (2005) Bach1 competes with Nrf2 leading to negative regulation of the antioxidant response element (ARE)mediated $\mathrm{NAD}(\mathrm{P}) \mathrm{H}$ :quinone oxidoreductase 1 gene expression and induction in response to antioxidants. J Biol Chem 280, 16891-16900

15. Oyake T, Itoh K, and Motohashi H et al (1996) Bach proteins belong to a novel family of BTB-basic leucine zipper transcription factors that interact with MafK and regulate transcription through the NF-E2 site. Mol Cell Biol 16, 6083-6095

16. Wang P, Peng X, Wei ZF et al (2015) Geraniin exerts cytoprotective effect against cellular oxidative stress by upregulation of Nrf2-mediated antioxidant enzyme expression via PI3K/AKT and ERK1/2 pathway. Biochim Biophys Acta 1850, 1751-1761

17. Zheng S, Huang Z and Zhang DD (2009) Phosphorylation of Nrf2 at multiple sites by MAP kinases has a limited contribution in modulating the Nrf2-dependent antioxidant response. PLoS One 4, e6588

18. Ling LT, Saito Y, Palanisamy UD, Cheng HM and Noriko $N$ (2012) Cytoprotective effects of geraniin against peroxynitrite- and peroxyl radical-induced cell death via free radical scavenging activity. Food Chem 132, 1899-1907

19. Kang KA, Lee IK, Zhang R et al (2011) Radioprotective effect of geraniin via the inhibition of apoptosis triggered by gamma-radiation-induced oxidative stress. Cell Biol Toxicol 27, 83-94

20. Beckman JD, Chen C, Nguyen J et al (2011) Regulation of heme oxygenase- 1 protein expression by miR-377 in combination with miR-217. J Biol Chem 286, 3194-3202

21. Mosmann T (1983) Rapid colorimetric assay for cellular growth and survival: application to proliferation and cytotoxicity assays. J Immunol Methods 65, 55-63

22. Mittal SPK, Kulkarni AP, Mathai J, Chattopadhyay S and Pal JK (2014) Dose-dependent differential response of mammalian cells to cytoplasmic stress is mediated through the heme-regulated elF2 $\alpha$ kinase. Int J Biochem Cell Biol 5, 186-197

23. Ohkawa H, Ohishi N and Yagi K (1979) Assay for lipid peroxides in animal tissues by thiobarbituric acid reaction. Anal Biochem 95, 351-358

24. Sohn JH, Han KL, Choo JH and Hwang JK (2007) Macelignan protects HepG2 cells against tert-butylhydroperoxide-induced oxidative damage. Biofactors 29, 1-10

25. Ellman GL (1959) Tissue sulfhydryl groups. Arch Biochem Biophys 82, 70-77

26. Kramer MF (2011) Stem-loop RT-qPCR for miRNAs. Curr Protoc Mol Biol Chapter 15, Unit 15, 10 